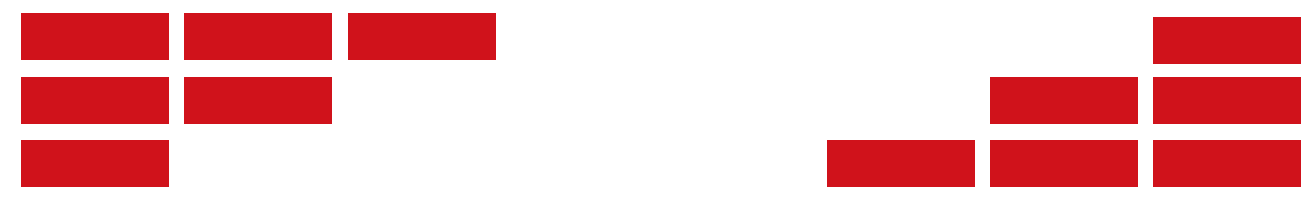

\title{
Impacto de las remesas \\ sobre la inclusión financiera: el caso de México ${ }^{1}$
}

\section{Impact of Remittances on Financial \\ Inclusion: the case of Mexico}

Rodrigo Carrillo

Universidad Iberoamericana Ciudad de México r.carrillovalles@gmail.com

Pablo Cotler*

Universidad Iberoamericana Ciudad de México pablo.cotler@ibero.mx

${ }^{1}$ Agradecemos los comentarios hechos por dos revisores.

* Autor para correspondencia. 


\section{Resumen}

Con base en datos municipales de México estudiamos si la recepción de remesas tiene un efecto sobre la inclusión financiera y su infraestructura. Para ello, utilizamos datos de panel y varias medidas de inclusión financiera, consideramos a los bancos y a las instituciones no bancarias, y usamos instrumentos basados en registros de residentes mexicanos en Estados Unidos. Encontramos que las remesas reducen el número de sucursales bancarias, pero aumentan el número de oficinas de las instituciones financieras no bancarias y de corresponsales. Además, las remesas aumentan el número de cuentas y de préstamos, con la excepción de los hipotecarios que otorga la banca. Estos impactos son mayores en la región sur del país, donde la pobreza y la exclusión financiera son más frecuentes.

Palabras clave: remesas, inclusión financiera, instituciones bancarias y no-bancarias.

\section{Abstract}

Using municipal data from Mexico we study whether the receipt of remittances has an effect on financial inclusion and its infrastructure. For such purpose, we use panel data, various measures of financial inclusion, consider banks and non-bank institutions and use instruments based on records of Mexican residents in the us. We find that remittances reduce the number of bank branches but increase the number offices of non-bank financial institutions and of correspondents. Further, remittances increase the number of accounts and of loans, with the exception of mortgages offered by banks. These impacts are greater in the southern region of the country, where poverty and financial exclusion are more prevalent.

Keywords: remittances, financial inclusion, banking and non-banking institutions.

JEL codes: F24, G21, O16

Fecha de recepción: 29 de septiembre de 2021.

Fecha de aceptación: 12 de noviembre de 2021. 


\section{Introducción}

México es uno de los países de ingreso medio que más remesas recibe del exterior. En 2020 llegaron 43 mil millones de dólares, de los cuales el 95\% fue enviado a través de transferencias electrónicas. El valor de la transferencia promedio fue de 340 dólares y según datos de la Encuesta Nacional de Ingreso y Gasto de los Hogares 2018, este flujo monetario fue recibido por el 5\% de la población mexicana. Considerando el impacto de esta entrada de dinero en las economías locales, es posible que las remesas pudieran ser una herramienta para elevar la inclusión financiera.

Una revisión de los estudios sobre el vínculo entre remesas e inclusión sugiere que no hay una respuesta concluyente. Como menciona Azizi (2020), los receptores de remesas pueden necesitar productos financieros para el resguardo de tales recursos. Pero, por otro lado, las remesas pueden relajar las restricciones financieras de los individuos y, con ello, reducir la demanda de productos de crédito. Así, dependiendo de qué tanta demanda haya por los productos financieros y qué rentabilidad se pueda obtener, los bancos decidirán si es conveniente establecer una sucursal o llevar sus servicios mediante corresponsales y cajeros. A este respecto, Demirgüç-Kunt et al. (2011) encuentran que mientras mayor sea el porcentaje de hogares perceptores de remesas, hay una mayor presencia de sucursales bancarias, y aumenta el número de cuentas y depósitos. Sin embargo, Martínez Pería et al. (2008) y Li Ng et al. (2015) no encuentran un impacto de las remesas sobre el uso de productos crediticios de la banca.

Pero en el caso de México, sólo 30\% de las remesas fueron cobradas a través de los bancos. El resto fue hecho a través de las entidades de ahorro y crédito popular (EACP) —instituciones no bancarias reguladas por la autoridad financiera-y de tiendas comerciales. En este sentido, para fines de evaluar el impacto de las remesas sobre la inclusión financiera consideramos necesario analizar que sucedió con el uso de los productos que ofrecen bancos y EACP, y con los puntos de venta de estos dos tipos de instituciones. ${ }^{2}$ En lugar de agregar los indicadores

2 Las EACP incluyen a las sociedades cooperativas de ahorro y préstamo y las sociedades financieras populares, ambas instituciones reguladas por la Comisión Nacional Bancaria de Valores. Para marzo de 2021 las primeras tenían ocho millones de socios, 2186 sucursales y activos por valor de 195 mil millones de pesos. Por otro lado, las sociedades financieras populares tenían cinco millones de clientes, 1112 sucursales y activos por valor de 32 mil millones de pesos. Los activos de las entidades de crédito y ahorro popular representan 
de bancos y EACP, optamos por mantenerlos separados para así investigar si las remesas causaban efectos diferenciados sobre la presencia institucional y el uso de sus productos financieros. Tal distinción obedece a que estos dos tipos de instituciones financieras son sustitutos imperfectos, pues la metodología para adquirir información no es similar, su población objetivo difiere en términos de ingreso, riqueza y formalidad, y el tamaño, la escala y la complejidad de los productos y los servicios que ofrecen también son muy distintos. En este sentido, la existencia o no de efectos diferenciados pudiera ser un insumo para futuros trabajos que deseen evaluar la calidad de la inclusión financiera - donde destacan las características de costo, transparencia, trato justo y amigable- (AFI, 2016).

Además de incorporar a las EACP, nuestra contribución a la discusión acerca del impacto que tienen las remesas sobre la inclusión financiera se encuentra en distintos rubros. En primer lugar, se utilizan datos al nivel municipal con una periodicidad trimestral. Por lo general, los trabajos publicados emplean datos de corte transversal. En segundo término, se incorporan distintas medidas de penetración y uso de productos y servicios financieros, lo cual permite tener una visión más integral de la inclusión financiera municipal. Además, ante un potencial problema de endogeneidad en las estimaciones, se propone el uso de características económicas del lugar desde donde se envían las remesas como variables instrumentales, ejercicio que no se ha hecho con datos de panel. Por último, además de utilizar mínimos cuadrados ordinarios también se estima un modelo de Poisson debido a la distribución que toman los valores de algunas variables dependientes.

Entre nuestros principales resultados tenemos que las remesas disminuyen la presencia de sucursales de la banca comercial y aumentan la presencia de corresponsales y de sucursales de las entidades de ahorro y crédito popular. Si bien los datos no permiten distinguir qué corresponsales atienden con exclusividad a los clientes de la banca, nuestras estimaciones sugieren que a diferencia de lo que acontece con las EACP, la presencia de sucursales bancarias reduce el número de corresponsales. En este sentido, el aumento de corresponsales y la disminución de sucursales bancarias pudiera ser parte de una estrategia de reducción de costos por parte de la banca. Por otro lado, aun controlando por la presencia de sucursales de bancos y de EACP, las remesas elevan el número de cajeros. En lo

el 2\% de los que tienen la banca. Por otro lado, el número de sucursales de las EACP es equivalente a un tercio de lo que tiene la banca. La correlación entre sucursales bancarias y sucursales de EACP por cada 10 mil adultos es de 0.057 . 
que se refiere al uso de productos financieros, se detecta - tanto para bancos como para $\mathrm{EACP}$ - que las remesas acarrean un incremento en el número de cuentas y en el crédito al consumo, siendo mayor el impacto para el caso de las EACP. En el caso del crédito relacionado con la vivienda, se halla que las remesas elevan los créditos que otorgan las EACP, pero reduce el que dan los bancos. Considerando que para conseguir uno de estos créditos se requiere acceder a sucursales, este pudiera ser una consecuencia de la estrategia seguida por los bancos respecto de dónde poner y quitar sucursales. Finalmente, si agrupamos a los municipios en cinco regiones geográficas se detecta que la zona sur — región en la cual es mayor la mayor tasa de pobreza y la exclusión financiera - es donde mayor impacto tienen las remesas sobre cuentas, crédito al consumo y puntos de acceso. Además, en dicha zona se observa una reducción de sucursales bancarias y un incremento de las oficinas de las EACP.

Para explicar dichos resultados, el artículo está dividido en cinco secciones adicionales. En la segunda presenta mos un breve repaso de las metodologías y los resultados hallados en otros trabajos. Posteriormente, en la tercera sección describimos los datos y la metodología que utilizamos. Luego en la cuarta sección presentamos los principales resultados y, finalmente, en la quinta concluimos.

\section{Revisión de la literatura}

La investigación sobre los efectos de las remesas en el crecimiento económico, pobreza, desigualdad, salud, educación, emprendimiento e inversión productiva es abundante. ${ }^{3}$ Sin embargo, existen pocos trabajos que abordan la causalidad de las remesas de origen internacional sobre la inclusión financiera. Es importante analizar esta relación pues existe bastante evidencia que sugiere que el uso adecuado de servicios financieros puede tener efectos positivos en el crecimiento económico y en la reducción de la pobreza (por ejemplo, Chibba, 2009; Kim, 2016).

Los trabajos empíricos sobre la relación entre remesas e inclusión financiera se pueden dividir según si los datos corresponden a una muestra de países o si se trata de los de un país en particular. Dentro de los primeros se encuentra el de Martínez Pería et al. (2008). Estos autores analizan si existe una causalidad entre la recepción de remesas y el desarrollo financiero, siendo este último definido por la evolución que toman los depósitos bancarios y el crédito al sector privado,

3 Para una amplia revisión véase Glystos (2005), Giuliano y Ruiz-Arranz (2009) y Demirgüç-Kunt et al. (2011). 
ambos como porcentaje del producto interno bruto (PIB). Utilizando datos de 18 países de América Latina para el período 1975-2001, los autores hallan que las remesas como proporción del PIB tienen un impacto positivo sobre ambos productos, siendo menor conforme la proporción de remesas recibidas a través de los bancos es menor.

Similar a los autores antes mencionados, Aggarwal et al. (2011) usan datos de flujos de remesas de 109 países, de 1975 a 2007, para indagar si estas afectan la evolución de los depósitos y los créditos que ofrece la banca como proporción del piв. En su trabajo se reconocen tres fuentes de endogeneidad: error de medición, causalidad inversa y variables omitidas. Para solucionar estos sesgos, recurren al Método Generalizado de Momentos (GMM) y a variables instrumentales correspondientes a diversas características del país emisor, entre las que se encuentran el PIB per cápita y la tasa de desempleo. Los autores concluyen que hay una causalidad positiva y significativa entre la recepción de remesas y el desarrollo financiero, especialmente en los países en desarrollo.

Si bien el uso de variables rezagadas como instrumentos puede solucionar el problema de causalidad inversa, no resuelven la endogeneidad por errores de medición, pues pueden ser también sujetos de dichos errores. Además, los resultados de esta metodología son sensibles a la elección del número de rezagos utilizados, lo que representa una dificultad en su implementación, particularmente cuando la muestra es grande en $T$ (Allison et al., 2017). Ante este inconveniente, Brown y Carmignani (2015) agregan los instrumentos en subgrupos. Utilizando datos de 1970 a 2009 de 135 países encuentran que la relación entre remesas y crédito bancario no es lineal. A niveles bajos de remesas, su recepción reduce el crédito bancario. Pero una vez que superan el $2.5 \%$ del PIB, el crédito aumenta con las remesas. Ben Naceur et al. (2020) llevan a cabo un ejercicio similar, con datos de 187 países entre 2004 y 2015, y encuentran que, a bajos niveles de remesas, estas funcionan como sustitutos de productos financieros formales, cuando superan el 13\% respecto al PIB, ellas tienden a aumentar la presencia de medios de acceso formales y el uso de los productos que estos ofrecen.

Además del estudio antes descrito, Martínez Pería et al. (2008) realizan un análisis de corte transversal para los casos de México y El Salvador. Entre las variables utilizadas para medir la inclusión financiera están la tenencia de cuentas bancarias, el crédito bancario y el acceso a sucursales de los bancos. Mientras que, a fin de atender el potencial problema de endogeneidad, para El Salvador, los autores utilizan características del lugar de origen de las remesas tales como el PIB, el PIB 
per cápita y la tasa de desempleo, y para México, la desviación de corto plazo en el nivel de precipitaciones y la cercanía a vías férreas que fueron puestas en los primeros ańos del siglo $\mathrm{xx}$ - previo a la primera gran ola migratoria hacia Estados Unidos- Para El Salvador los resultados indican que los hogares receptores de remesas son más propensos a abrir cuentas bancarias, mientras que, para México, en aquellos municipios donde una mayor parte de los hogares reporta recibir remesas, hay más depósitos, cuentas y sucursales en términos per cápita. Para ambos países no se encuentran indicios de que las remesas tengan un impacto sobre los productos de crédito. En Demirgüç-Kunt et al. (2011) se extiende el análisis de Martínez Pería et al (2008) para el caso mexicano al utilizar un modelo Tobit, pues un número significativo de los municipios reportan variables dependientes igual a cero. Entre sus diversos resultados, sobresale que donde hay un mayor porcentaje de hogares que reportan recibir remesas, hay también una mayor presencia de sucursales bancarias, un mayor número de cuentas per cápita y los depósitos como porcentaje del PIB son mayores.

En contraposición a dicha metodología, Ambrosius (2011) opta por solo observar lo que ocurre en los municipios donde hay presencia financiera y utiliza mínimos cuadrados ordinarios, argumentando que incluso si un municipio recibe grandes montos de remesas, esto por sí solo no hará que se ofrezcan servicios financieros, particularmente si se trata de pequeñas poblaciones o lugares alejados geográficamente. A causa de este sesgo en la estimación, los resultados son exclusivos para los municipios con presencia financiera. Con datos de El Salvador entre 2007 y 2010 encuentra que, en aquellos municipios donde una proporción mayor de hogares recibe remesas del ex terior hay también más ahorros y nú mero de cuentas per cápita. Utilizando una metodología y una muestra distinta de datos, Anzoátegui et al. (2014) llegan a un resultado similar, pero señalan además que las remesas no afectan el uso de productos de crédito. Para ello utilizan datos de hogares de 1995 a 2001 y un modelo probit que incorpora como instrumentos al piв y al porcentaje de pobreza al nivel estatal del lugar de residencia del familiar emisor de la remesa.

\section{Datos y metodología}

En consideración de la posible endogeneidad proveniente por causalidad inversa o por correlación de la variable remesas con el término de error optamos por el uso de estimaciones con variables instrumentales. Similar a Martínez Pería et al. 
(2008), utilizamos dos instrumentos referentes a las características económicas del lugar emisor de la remesa: PIB per cápita y tasa de desempleo, ambos datos tomados de la Oficina de Análisis Económico de Estados Unidos. La información utilizada como instrumento es el promedio registrado en el lugar de origen dentro de Estados Unidos — país de donde llega el 95.3\% de las remesas- ponderada por la presencia de población inmigrante. Para la estimación de estos ponderadores es necesario conocer de qué municipio mexicano salieron los migrantes que envían remesas y en qué lugar de Estados Unidos viven, información que se obtuvo de los datos de matrículas consulares emitidas por la Secretaría de Relaciones Exteriores del Gobierno de México durante 2010 y 2019. ${ }^{4}$ La Figura 1 muestra el origen-destino de la población mexicana en Estados Unidos.

Figura 1: Municipios de origen de población mexicana en Estados Unidos por principal división de residencia, 2010-2019

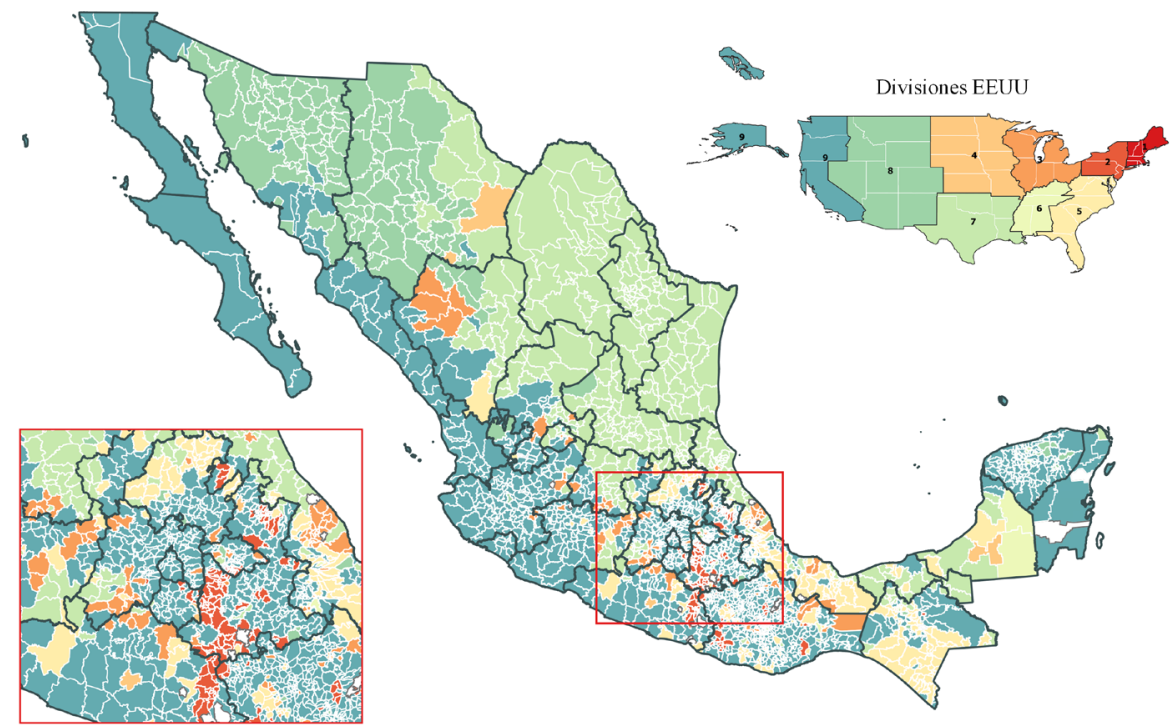

Fuente: Elaboración propia con base en los Registros de Matrículas Consulares (SRE).

4 Durante los años referidos se emitieron alrededor de 886 mil matrículas consulares a mexicanos en Estados Unidos. 
Ante las diversas fuentes de endogeneidad, en el trabajo empírico es común el uso de distintas estimaciones GMM, pero estas presentan inconvenientes cuando $T$ y $N$ tienden a ser grandes, como es el caso de nuestra muestra al nivel municipal que abarca el periodo 2013-2020.5 Por ello, optamos por estimaciones de mínimos cuadrados ordinarios con efectos fijos y efectos aleatorios, y donde en ambos casos instrumentamos a la variable remesas con las dos variables antes mencionadas, tal que:

$$
\log \left(I F_{i t}\right)=\beta_{1} \log \left(\operatorname{Rem}_{i t}\right)+\beta_{2}^{\prime} X_{i t}+\alpha_{i}+u_{i t}
$$

donde $I F$ se refiere a las distintas medidas de inclusión financiera, ${ }^{6}$ cuyos datos provienen de los reportes trimestrales de la Comisión Nacional Bancaria y de Valores. El último reporte disponible al momento de la elaboración de este trabajo es del segundo trimestre de 2020. Rem es el monto de remesas recibidas al nivel municipal, publicado por el Banco de México desde 2013. Por tanto, la temporalidad estudiada abarca del primer trimestre de 2013 al segundo de 2020. Además, $X$ contiene controles como logaritmo de la población —dato también incluido en los reportes de inclusión financiera-y el promedio años de escolaridad al nivel municipal, calculado con datos del Censo de Población 2010 y la Encuesta Intercensal 2015 que elabora el Instituto Nacional de Estadística y Geografía (INEGI). Como aproximación del dinamismo económico al nivel municipal utilizamos el logaritmo del consumo eléctrico per cápita anual de la Comisión Federal de Electricidad. ${ }^{7}$

5 Una posible alternativa para una muestra con estas características puede ser la propuesta por Hsiao (2007). Sin embargo, también descartamos esta metodología pues dicha propuesta asume que los regresores son exógenos, lo cual no ocurre con nuestra variable explicativa remesas.

6 Estas incluyen variables de acceso como: sucursales de la banca comercial, sucursales de las sociedades cooperativas de ahorro y préstamo, corresponsales y cajeros automáticos. En lo que se refiere a productos bancarios, consideramos número de cuentas, créditos hipotecarios y créditos al consumo. Y para el caso de las EACP se consideran los depósitos de ahorro y a la vista, los créditos a la vivienda y los créditos al consumo. La información sobre corresponsales, cajeros automáticos y transacciones en cajeros automáticos no permite distinguir si estos corresponden a bancos o a las EACP.

7 Otras opciones consideradas fueron la principal actividad económica e incidencia de pobreza. Sin embargo, optamos por consumo eléctrico porque 
En caso de que la variable dependiente mida el acceso a servicios financieros, en $X$ se incluye el logaritmo de sucursales bancarias o corresponsales para medir la competencia existente. Por otro lado, cuando la variable dependiente se refiere al uso de productos, el logaritmo de sucursales busca medir la cobertura de las instituciones que los ofrecen. Y, finalmente, en caso de que la variable dependiente sea un producto de crédito, en $X$ se incorporan además variables dicotómicas que indican el trimestre al que corresponde $t$, bajo el supuesto de que la demanda por productos crediticios pudiera presentar alguna estacionalidad.

Dado que algunas observaciones de las variables incluidas en $I F$ toman el valor de cero, las estimaciones utilizan — similar a Ambrosius (2011) — una muestra truncada. En la búsqueda de aprovechar estos registros nulos y verificar la robustez de nuestros resultados, estimamos adicionalmente un modelo Poisson que incorpora una función control con efectos fijos para corregir endogeneidad, como sugieren Lin y Wooldridge (2019), tal que: ${ }^{8}$

$$
I F_{i t}=\beta_{1} \log \left(\operatorname{Rem}_{i t}\right)+\beta_{2}^{\prime} X_{i t}+\hat{\ddot{u}}_{i t}+v_{i t}
$$

donde $\hat{\ddot{u}}_{i t}$ es el término de error estimado en:

$$
\log \left(R \ddot{e} m_{i t}\right)=\beta_{1}^{\prime} \ddot{X}_{i t}+\ddot{u}_{i t}
$$

Por último, en consideración de posibles efectos locales, en la sección de resultados hacemos un comparativo del efecto de la variable Rem por regiones para las estimaciones descritas. Estas regiones se definieron siguiendo la clasificación utilizada por el Centro de Estudios Espinosa Yglesias (CEEY). ${ }^{?}$

los datos son publicados con mayor frecuencia y tienen un comportamiento similar. Por ejemplo, la correlación entre pobreza y consumo eléctrico en 2010 es del -0.69 y significativa al $95 \%$.

8 Se consideró también hacer una regresión binomial negativa. Sin embargo, este tipo de estimación puede tener un desempeño inadecuado cuando se consideran efectos fijos, pues existe la posibilidad de obtener un coeficiente distinto a cero en un regresor que no varía a través del tiempo. Aun así, se hicieron estimaciones con dicha metodología y se encontraron resultados que son similares en términos de dirección y significancia a los estimados con Poisson.

9 Las regiones están compuestas de la siguiente manera: $i$ ) Norte: Baja California, Sonora, Chihuahua, Nuevo León y Tamaulipas, ii) Norte-Occidente: Baja California Sur, Sinaloa, Nayarit, Durango y Zacatecas, iii) Centro-Norte: Jalisco, Aguascalientes, Colima, Michoacán y San Luis Potosí, iv)Centro: Guanajuato, Querétaro, 


\section{Resultados}

Con el fin de evaluar la relevancia de los dos instrumentos se recurrió a la prueba de Wald. En la Tabla 1 se observa que, en general, el logaritmo del ingreso per cápita de la entidad de donde provienen las remesas tiene un mejor desempeño como instrumento. Solo para el caso de créditos hipotecarios ofrecidos por la banca comercial no se identifica un instrumento de utilidad. Ante ello, en lo que sigue únicamente se presentan los resultados cuando se utiliza el logaritmo del ingreso per cápita.

\section{Tabla 1: Wald-test de las variables instrumentales}

Variable dependiente

\begin{tabular}{|c|c|c|c|c|c|}
\hline \multicolumn{2}{|c|}{ Variable dependiente } & \multirow{2}{*}{$\begin{array}{c}\text { Log. PIB } \\
\text { per cápita } \\
7.39^{* * *}\end{array}$} & \multirow{2}{*}{$\begin{array}{c}\text { Desempleo } \\
3.02^{*}\end{array}$} & \multirow{2}{*}{$\begin{array}{c}\text { Log. PIB } \\
\text { per cápita } \\
6.14^{* *}\end{array}$} & \multirow{2}{*}{$\begin{array}{c}\text { Desempled } \\
5.56^{* *}\end{array}$} \\
\hline \multirow{5}{*}{$\begin{array}{l}\text { Acceso a } \\
\text { servicios } \\
\text { financieros }\end{array}$} & Sucursales Banca Comercial & & & & \\
\hline & Sucursales EACP & $19.2 .8^{* * *}$ & $16.94^{* * *}$ & $12.14^{* * *}$ & $4.97^{* *}$ \\
\hline & Corresponsales bancarios & $271.73^{* *}$ & $191.13^{* *}$ & $58.66^{* * *}$ & $43.30^{* * *}$ \\
\hline & Cajeros automáticos & $124.98^{* * *}$ & $101.44^{* *}$ & $14.33^{* * *}$ & $11.93^{* * *}$ \\
\hline & $\begin{array}{l}\text { Núm. De transacciones en } \\
\text { cajeros automáticos }\end{array}$ & $5.60^{* *}$ & 2.15 & $22.11^{* * *}$ & $13.75^{* * *}$ \\
\hline \multirow{3}{*}{$\begin{array}{l}\text { Uso de } \\
\text { productos } \\
\text { bancarios }\end{array}$} & Cuentas & $60.30^{* * *}$ & 0.26 & 1.96 & $3.34^{*}$ \\
\hline & Créditos hipotecarios & 0.42 & 0.08 & 2.15 & 2.30 \\
\hline & Créditos al consumo & $70.67^{* * *}$ & $192.94^{* * *}$ & $7.11^{*}$ & $7.33^{* * *}$ \\
\hline \multirow{3}{*}{$\begin{array}{l}\text { Uso de } \\
\text { productos } \\
\text { EACP }\end{array}$} & $\begin{array}{l}\text { Depósitos al ahorro y a la } \\
\text { vista }\end{array}$ & 114.09 & 99.64 & $52.92^{* * *}$ & $43.78^{* * *}$ \\
\hline & Créditos a la vivienda & 0.22 & 0.69 & $8.77^{* * *}$ & $4.23^{* *}$ \\
\hline & Créditos al consumo & $32.21^{* * *}$ & $42.64^{* * *}$ & $41.39^{* * *}$ & $6.67^{* * *}$ \\
\hline \multicolumn{6}{|c|}{$\mathrm{p}<0.01,{ }^{* *} \mathrm{p}<0.05,{ }^{*} \mathrm{p}<0.1 . \mathrm{H} 0: \beta=0$} \\
\hline \multicolumn{6}{|c|}{$\begin{array}{l}\text { Hidalgo, Estado de México, Ciudad de México, Morelos, Tlaxcala y Puebla, } \\
\text { y v) Sur: Guerrero, Oaxaca, Chiapas, Veracruz, Tabasco, Campeche, Yucatán } \\
\text { y Quintana Roo. De acuerdo con estimaciones hechas con datos del Consejo } \\
\text { Nacional de Evaluación de la Política de Desarrollo Social (Coneval), en } 2018 \\
\text { la incidencia de pobreza en cada una de estas regiones era de } 24.2,34.6,35.4 \text {, } \\
42.6 \text { y } 60.9 \% \text {, respectivamente. Optamos por esta clasificación porque, de acuer- } \\
\text { do con el cEey, las regiones están definidas de modo que las entidades que las } \\
\text { conforman tienen características en términos de desigualdad. }\end{array}$} \\
\hline
\end{tabular}


En la Tabla 2 se presentan los resultados para los casos en que la variable dependiente describe el nivel de acceso a servicios financieros. En cuanto a la principal variable de interés, se encuentra que a mayores montos de remesas aumenta el número de sucursales de las EACP, hay más corresponsales, un mayor número de cajeros automáticos pero una menor cantidad de sucursales bancarias. Si bien los datos no permiten distinguir qué corresponsales atienden con exclusividad a los clientes de la banca, la estimación Poisson sugiere que, a diferencia de lo que acontece con las EACP, la presencia de sucursales bancarias reduce el número de corresponsales. ${ }^{10}$ En este sentido, el aumento de corresponsales y la reducción de sucursales bancarias pudiera ser parte de una estrategia de reducción de costos por parte de la banca. Por otro lado, aun controlando por la presencia de sucursales de ambos tipos de instituciones financieras, las remesas elevan el número de cajeros y también el número de transacciones en ellos.

En lo que se refiere al uso de productos financieros, en la Tablas 3 y 4 se detecta que, independientemente del tipo de institución, las remesas acarrean un incremento en el número de cuentas y en el crédito al consumo. ${ }^{11}$ En cuanto a los productos de crédito relacionados con la vivienda, los resultados no son concluyentes, pues depende del modelo empírico utilizado. En caso de utilizar mínimos cuadrados con efectos fijos, el impacto de las remesas sobre estos productos es nulo para ambos tipos de instituciones financieras. Sin embargo, ante el bajo uso de estos productos en los municipios donde se reciben remesas, quizás sea más conveniente recurrir a un modelo de Poisson. En dicho caso, los resultados sugieren que las remesas impactan positivamente sobre los productos de crédito que ofrecen las EACP y negativamente en el caso de los que ofrece la banca. Aun cuando hay diversas posibles razones para este resultado, es importante considerar que para la obtención de este tipo de crédito es necesario ir a una sucursal. En este sentido, el impacto negativo que tienen las remesas sobre las sucursales de la banca es consistente con el efecto negativo que tienen sobre el crédito hipotecario. De igual manera, el impacto positivo que tienen las remesas sobre las sucursales de las EACP es consistente con la causalidad positiva sobre el crédito a la vivienda.

${ }^{10}$ El impacto positivo que tienen las remesas sobre las oficinas de EACP corresponde al comportamiento mostrado por las sociedades cooperativas de ahorro y préstamo.

${ }^{11}$ El crédito al consumo integra los préstamos personales, los préstamos de nómina y los créditos $\mathrm{ABCD}$. Si se hacen estimaciones individuales para cada tipo de crédito los resultados son similares. 
Tabla 2: Resultados para variables de acceso a servicios financieros

\begin{tabular}{|c|c|c|c|c|c|c|c|c|c|c|}
\hline \multirow[t]{2}{*}{$\begin{array}{l}\text { IV: Log. PIB per } \\
\text { cápita }\end{array}$} & \multicolumn{2}{|c|}{$\begin{array}{l}\text { Sucursales banca } \\
\text { comercial }\end{array}$} & \multicolumn{2}{|c|}{ Sucursales EACP } & \multicolumn{2}{|c|}{$\begin{array}{l}\text { Corresponsales } \\
\text { bancarios }\end{array}$} & \multicolumn{2}{|c|}{ Cajeros automáticos } & \multicolumn{2}{|c|}{$\begin{array}{l}\text { No. Transacciones } \\
\text { en cajeros } \\
\text { automáticos }\end{array}$} \\
\hline & FE & Poisson & FE & Poisson & FE & Poisson & FE & Poisson & FE & Poisson \\
\hline Log. Remesas & $\begin{array}{l}-0.100^{* *} \\
(0.037)\end{array}$ & $\begin{array}{l}-0.105^{* *} \\
(0.037)\end{array}$ & $\begin{array}{c}0.314^{* * *} \\
(0.071)\end{array}$ & $\begin{array}{l}0.445^{* *} \\
(0.144)\end{array}$ & $\begin{array}{l}1.532^{* * *} \\
(0.093)\end{array}$ & $\begin{array}{c}0.799^{* * *} \\
(0.066)\end{array}$ & $\begin{array}{c}0.929^{* * *} \\
(0.083)\end{array}$ & $\begin{array}{c}0.655^{* * *} \\
(0.084)\end{array}$ & $\begin{array}{c}0.430^{* * *} \\
(0.086)\end{array}$ & $\begin{array}{r}0.994^{* * *} \\
(0.183)\end{array}$ \\
\hline Log. Población & $\begin{array}{c}0.218 \\
(0.190)\end{array}$ & $\begin{array}{l}0.368^{* *} \\
(0.122)\end{array}$ & $\begin{array}{c}0.155 \\
(0.367)\end{array}$ & $\begin{array}{c}0.267 \\
(0.416)\end{array}$ & $\begin{array}{c}0.366 \\
(0.368)\end{array}$ & $\begin{array}{l}1.033^{* *} \\
(0.386)\end{array}$ & $\begin{array}{l}-0.887^{*} \\
(0.448)\end{array}$ & $\begin{array}{l}-0.552 \\
(0.555)\end{array}$ & $\begin{array}{l}0.756^{*} \\
(0.317)\end{array}$ & $\begin{array}{l}-0.154 \\
(0.433)\end{array}$ \\
\hline Escolaridad & $\begin{array}{c}0.022 \\
(0.013)\end{array}$ & $\begin{array}{l}-0.017^{*} \\
(0.008)\end{array}$ & $\begin{array}{l}-0.060^{* *} \\
(0.023)\end{array}$ & $\begin{array}{l}-0.042 \\
(0.039)\end{array}$ & $\begin{array}{c}0.169^{* * *} \\
(0.041)\end{array}$ & $\begin{array}{c}0.301^{* * *} \\
(0.024)\end{array}$ & $\begin{array}{c}0.086^{* * *} \\
(0.025)\end{array}$ & $\begin{array}{l}0.078^{* *} \\
(0.028)\end{array}$ & $\begin{array}{l}-0.036 \\
(0.024)\end{array}$ & $\begin{array}{l}-0.039 \\
(0.024)\end{array}$ \\
\hline $\begin{array}{l}\text { Log. Consumo } \\
\text { eléctrico }\end{array}$ & $\begin{array}{c}0.017 \\
(0.009)\end{array}$ & $\begin{array}{c}0.019 \\
(0.011)\end{array}$ & $\begin{array}{c}0.008 \\
(0.013)\end{array}$ & $\begin{array}{l}-0.008 \\
(0.016)\end{array}$ & $\begin{array}{c}0.019 \\
(0.026)\end{array}$ & $\begin{array}{c}0.007 \\
(0.020)\end{array}$ & $\begin{array}{l}-0.005 \\
(0.024)\end{array}$ & $\begin{array}{l}-0.005 \\
(0.021)\end{array}$ & $\begin{array}{l}-0.004 \\
(0.015)\end{array}$ & $\begin{array}{l}-0.010 \\
(0.014)\end{array}$ \\
\hline $\begin{array}{l}\text { Log. Sucursales } \\
\text { banca comercial }\end{array}$ & & & & & $\begin{array}{l}-0.104 \\
(0.061)\end{array}$ & $\begin{array}{c}-0.174^{* * *} \\
(0.048)\end{array}$ & $\begin{array}{l}0.099^{*} \\
(0.047)\end{array}$ & $\begin{array}{l}0.171^{* *} \\
(0.056)\end{array}$ & & \\
\hline $\begin{array}{l}\text { Log. Sucursales } \\
\text { EACP }\end{array}$ & & & & & $\begin{array}{l}-0.037 \\
(0.031)\end{array}$ & $\begin{array}{c}0.022 \\
(0.023)\end{array}$ & $\begin{array}{l}-0.004 \\
(0.019)\end{array}$ & $\begin{array}{l}-0.029 \\
(0.021)\end{array}$ & & \\
\hline $\begin{array}{l}\text { Log. } \\
\text { Corresponsales }\end{array}$ & $\begin{array}{l}-0.006 \\
(0.009)\end{array}$ & $\begin{array}{l}-0.013 \\
(0.009)\end{array}$ & $\begin{array}{c}0.000 \\
(0.014)\end{array}$ & $\begin{array}{c}0.000 \\
(0.021)\end{array}$ & & & & & & \\
\hline Constante & $\begin{array}{l}-1.110 \\
(1.987)\end{array}$ & & $\begin{array}{l}-1.128 \\
(3.689)\end{array}$ & & $\begin{array}{l}-6.368 \\
(3.833)\end{array}$ & & $\begin{array}{l}9.375^{*} \\
(4.616)\end{array}$ & & $\begin{array}{l}-0.010 \\
(3.094)\end{array}$ & \\
\hline $\begin{array}{l}\text { Efectos } \\
\text { temporales }\end{array}$ & No & No & No & No & No & No & No & No & No & No \\
\hline Observaciones & 25,306 & 26,283 & 22,158 & 26,210 & 17,849 & 18,101 & 17,850 & 18,064 & 37,303 & 38,271 \\
\hline Municipios & 973 & 973 & 1,009 & 1,006 & 758 & 743 & 757 & 741 & 1,532 & 1,519 \\
\hline $\mathrm{R}^{2}$ Total & 0.546 & & 0.479 & & 0.492 & & 0.003 & & 0.117 & \\
\hline
\end{tabular}

${ }^{* * *} \mathrm{p}<0.01,{ }^{* *} \mathrm{p}<0.05,{ }^{*} \mathrm{p}<0.1$. Errores estándar entre paréntesis. En todos los casos se rechazó la hipótesis nula del test de Hausman, por lo que solo se presentan los resultados de FE. 
Tabla 3: Resultados para variables de uso de productos de banca comercial

\begin{tabular}{|c|c|c|c|c|c|c|}
\hline \multirow[t]{2}{*}{ IV: Log. PIB per cápita } & \multicolumn{2}{|c|}{ Cuentas } & \multicolumn{2}{|c|}{ Créditos hipotecarios } & \multicolumn{2}{|c|}{ Créditos al consumo } \\
\hline & FE & Poisson & FE & Poisson & FE & Poisson \\
\hline \multirow[t]{2}{*}{ Log. Remesas } & $0.355^{* * *}$ & -0.599 & -0.089 & $-0.912^{* * *}$ & $0.564^{* * *}$ & $0.633^{*}$ \\
\hline & $(0.046)$ & $(0.315)$ & $(0.138)$ & $(0.214)$ & $(0.067)$ & $(0.256)$ \\
\hline \multirow[t]{2}{*}{ Log. Población } & -0.123 & $3.542^{*}$ & $3.045^{* * *}$ & $9.614^{* * *}$ & $1.334^{* * *}$ & 0.222 \\
\hline & $(0.188)$ & $(1.618)$ & $(0.774)$ & $(1.488)$ & $(0.377)$ & $(1.144)$ \\
\hline \multirow[t]{2}{*}{ Escolaridad } & $-0.155^{* * *}$ & $-0.111^{*}$ & $0.087^{* *}$ & -0.091 & $0.257^{* * *}$ & -0.104 \\
\hline & $(0.017)$ & $(0.045)$ & $(0.034)$ & $(0.131)$ & $(0.020)$ & $(0.376)$ \\
\hline \multirow[t]{2}{*}{ Log. Consumo eléctrico } & $0.038^{* *}$ & -0.062 & -0.042 & -0.239 & -0.001 & -0.041 \\
\hline & $(0.015)$ & $(0.072)$ & $(0.037)$ & $(0.131)$ & $(0.013)$ & $(0.083)$ \\
\hline \multirow{2}{*}{$\begin{array}{l}\text { Log. Sucursales banca } \\
\text { comercial }\end{array}$} & $0.067^{*}$ & -0.227 & $0.128^{*}$ & -0.075 & $0.083^{* *}$ & 0.299 \\
\hline & $(0.030)$ & $(0.130)$ & $(0.061)$ & $(0.154)$ & $(0.029)$ & $(0.158)$ \\
\hline \multirow[t]{2}{*}{ Constante } & $10.841^{* * *}$ & & $-27.408^{* * *}$ & & $-8.676^{*}$ & \\
\hline & $(1.921)$ & & $(7.840)$ & & $(3.817)$ & \\
\hline Efectos temporales & No & No & Sí & Sí & Sí & Sí \\
\hline Observaciones & 26,167 & 26,162 & 25,965 & 26,151 & 26,189 & 26,185 \\
\hline Municipios & 986 & 981 & 983 & 979 & 986 & 982 \\
\hline $\mathrm{R}^{2}$ Total & 0.161 & & 0.693 & & 0.795 & \\
\hline
\end{tabular}

${ }^{* * *} \mathrm{p}<0.01,{ }^{* *} \mathrm{p}<0.05,{ }^{*} \mathrm{p}<0.1$. Errores estándar entre paréntesis. En todos los casos se rechazó la hipótesis nula del test de Hausman, por lo que solo se presentan los resultados de FE. 
Tabla 4: Resultados para variables de uso de productos de Entidades de Ahorro y Crédito Popular (EACP)

\begin{tabular}{|c|c|c|c|c|c|c|}
\hline \multirow{2}{*}{ IV: Log. PIB per cápita } & \multicolumn{2}{|c|}{ Depósitos al ahorro y a la vista } & \multicolumn{2}{|c|}{ Créditos a la vivienda } & \multicolumn{2}{|c|}{ Créditos al consumo } \\
\hline & FE & Poisson & FE & Poisson & FE & Poisson \\
\hline \multirow[t]{2}{*}{ Log. Remesas } & $1.675^{* * *}$ & $0.947^{* * *}$ & -0.152 & $4.462^{* * *}$ & $0.899^{* * *}$ & 0.258 \\
\hline & $(0.157)$ & $(0.134)$ & $(0.323)$ & $(0.559)$ & $(0.156)$ & $(0.151)$ \\
\hline \multirow[t]{2}{*}{ Log. Población } & $1.369^{*}$ & $1.421^{*}$ & $-5.769^{* * *}$ & 1.515 & 0.683 & 0.170 \\
\hline & $(0.672)$ & $(0.663)$ & $(1.736)$ & $(2.263)$ & $(0.735)$ & $(0.862)$ \\
\hline \multirow[t]{2}{*}{ Escolaridad } & $0.175^{* *}$ & -0.029 & $0.261^{* *}$ & $-1.839^{* * *}$ & 0.038 & -0.072 \\
\hline & $(0.062)$ & $(0.050)$ & $(0.087)$ & $(0.164)$ & $(0.062)$ & $(0.108)$ \\
\hline \multirow[t]{2}{*}{ Log. Consumo eléctrico } & 0.055 & -0.016 & -0.015 & 0.147 & 0.003 & -0.018 \\
\hline & $(0.038)$ & $(0.019)$ & $(0.081)$ & $(0.075)$ & $(0.036)$ & $(0.028)$ \\
\hline \multirow[t]{2}{*}{ Log. Sucursales EACP } & $0.320^{* * *}$ & $0.361^{* * *}$ & $0.426^{* * *}$ & -0.065 & $0.425^{* * *}$ & $0.396^{* * *}$ \\
\hline & $(0.065)$ & $(0.051)$ & $(0.089)$ & $(0.124)$ & $(0.069)$ & $(0.050)$ \\
\hline \multirow[t]{2}{*}{ Constante } & -11.467 & & $62.347^{* * *}$ & & -2.669 & \\
\hline & $(6.580)$ & & $(17.732)$ & & $(7.287)$ & \\
\hline Efectos temporales & No & No & Sí & Sí & Sí & Sí \\
\hline Observaciones & 24,595 & 24,577 & 16,279 & 24,486 & 24,437 & 24,573 \\
\hline Municipios & 1,101 & 1,036 & 927 & 1,020 & 1,094 & 1,035 \\
\hline $\mathrm{R}^{2}$ Total & 0.288 & & 0.033 & & 0.287 & \\
\hline
\end{tabular}

${ }^{* * *} \mathrm{p}<0.01,{ }^{* *} \mathrm{p}<0.05,{ }^{*} \mathrm{p}<0.1$. Errores estándar entre paréntesis. En todos los casos se rechazó la hipótesis nula del test de Hausman, por lo que solo se presentan los resultados de FE. 
Tabla 5: Coeficientes beta de Remesas por región

\begin{tabular}{|c|c|c|c|c|c|c|c|c|c|c|}
\hline \multirow{2}{*}{$\begin{array}{l}\text { IV: Log. PIB per } \\
\text { cápita }\end{array}$} & \multicolumn{2}{|c|}{ Norte } & \multicolumn{2}{|c|}{ Norte-Occidente } & \multicolumn{2}{|c|}{ Centro-Norte } & \multicolumn{2}{|c|}{ Centro } & \multicolumn{2}{|c|}{ Sur } \\
\hline & FE/RE & Poisson & FE/RE & Poisson & FE/RE & Poisson & FE/RE & Poisson & FE/RE & Poisson \\
\hline \multirow{2}{*}{$\begin{array}{l}\text { Sucursales banca } \\
\text { comercial }\end{array}$} & $-0.18^{* * *}$ & -0.002 & -0.062 & -0.006 & $-0.111^{* *}$ & $-0.01^{* * *}$ & -0.133 & -0.003 & $-0.170^{* *}$ & -0.012 \\
\hline & $(0.053)$ & $(0.124)$ & $(0.136)$ & $(0.083)$ & (0.048) & $(0.053)$ & $(0.094)$ & $(0.068)$ & $(0.063)$ & $(0.136)$ \\
\hline \multirow[t]{2}{*}{ Sucursales EACP } & 0.177 & 0.069 & -0.192 & -0.290 & $0.440^{* * *}$ & $0.088^{* *}$ & $0.476^{* * *}$ & $0.083^{*}$ & $0.804^{* * *}$ & $0.501^{* * *}$ \\
\hline & $(0.164)$ & $(0.634)$ & $(0.170)$ & $(1.474)$ & $(0.076)$ & $(0.150)$ & $(0.094)$ & $(0.262)$ & $(0.133)$ & $(0.240)$ \\
\hline \multirow[t]{2}{*}{ Corresponsales } & $0.538^{* * *}$ & 0.001 & $0.566^{* *}$ & $0.008^{* *}$ & $0.632^{* * *}$ & $0.005^{*}$ & $1.617^{* * *}$ & $0.013^{* * *}$ & $1.875^{* * *}$ & $0.022^{* * *}$ \\
\hline & $(0.081)$ & $(0.116)$ & $(0.244)$ & $(0.227)$ & $(0.197)$ & $(0.219)$ & $(0.421)$ & (0.119) & $(0.262)$ & $(0.231)$ \\
\hline \multirow[t]{2}{*}{ Cajeros automáticos } & $0.152^{* * *}$ & $0.002^{* * *}$ & $0.541^{* * *}$ & $0.01^{* * *}$ & $0.757^{* * *}$ & $0.006^{* * *}$ & $0.719^{* * *}$ & $0.005^{* * *}$ & $0.894^{* * *}$ & $0.009^{* * *}$ \\
\hline & $(0.056)$ & $(0.080)$ & $(0.213)$ & $(0.124)$ & $(0.179)$ & $(0.104)$ & $(0.213)$ & $(0.187)$ & $(0.187)$ & $(0.149)$ \\
\hline \multirow{2}{*}{$\begin{array}{l}\text { Trans. en cajeros } \\
\text { automáticos }\end{array}$} & $1.307^{* * *}$ & $0.001^{* *}$ & 0.412 & 0.000 & 0.172 & -0.000 & 0.821 & $0.001^{* *}$ & $1.392^{* * *}$ & $0.002^{* * *}$ \\
\hline & (0.192) & $(0.478)$ & $(0.162)$ & $(0.762)$ & $(0.177)$ & $(0.231)$ & $(0.268)$ & $(0.503)$ & $(0.154)$ & $(0.448)$ \\
\hline \multirow[t]{2}{*}{ Cuentas } & -0.082 & -0.000 & 0.059 & $-0.000^{*}$ & $0.191^{* *}$ & -0.000 & $0.256^{*}$ & -0.000 & $0.479^{* * *}$ & 0.000 \\
\hline & $(0.147)$ & $(0.139)$ & $(0.143)$ & $(0.483)$ & $(0.109)$ & $(0.115)$ & $(0.167)$ & $(0.918)$ & $(0.081)$ & $(0.151)$ \\
\hline \multirow{2}{*}{$\begin{array}{l}\text { Créditos } \\
\text { hipotecarios }\end{array}$} & -0.443 & $-0.00^{* * *}$ & $-0.595^{* *}$ & 0.000 & $-0.43^{* * *}$ & -0.000 & -0.052 & $-0.00^{* * *}$ & $0.201^{*}$ & $0.000^{*}$ \\
\hline & $(0.428)$ & $(0.144)$ & $(0.453)$ & $(0.218)$ & $(0.272)$ & $(0.207)$ & $(0.335)$ & $(0.296)$ & $(0.143)$ & $(0.262)$ \\
\hline \multirow{2}{*}{$\begin{array}{l}\text { Créditos al } \\
\text { consumo }\end{array}$} & $-0.252^{* *}$ & $-0.000^{*}$ & $0.459^{* * *}$ & $0.00^{* * *}$ & $0.200^{* *}$ & $0.000^{*}$ & $0.746^{* * *}$ & $0.000^{* * *}$ & $0.710^{* * *}$ & $0.000^{* *}$ \\
\hline & $(0.142)$ & $(0.802)$ & $(0.212)$ & $(0.145)$ & $(0.120)$ & $(0.077)$ & $(0.164)$ & $(0.301)$ & $(0.084)$ & $(0.091)$ \\
\hline \multirow{2}{*}{$\begin{array}{l}\text { Depósitos al ahorro } \\
\text { y a la vista }\end{array}$} & $1.246^{* * *}$ & $0.000^{* *}$ & $1.515^{* *}$ & 0.000 & -0.075 & $0.000^{*}$ & $2.020^{* * *}$ & $0.000^{* * *}$ & $2.288^{* * *}$ & $0.000^{* * *}$ \\
\hline & $(0.387)$ & $(0.246)$ & $(0.930)$ & $(0.576)$ & $(0.268)$ & $(0.262)$ & $(0.540)$ & $(0.317)$ & $(0.439)$ & $(0.232)$ \\
\hline \multirow{2}{*}{$\begin{array}{l}\text { Créditos a la } \\
\text { vivienda }\end{array}$} & -0.317 & 0.000 & 1.010 & $0.002^{* * *}$ & $-1.24^{* * *}$ & $0.001^{*}$ & 0.503 & $0.001^{* * *}$ & 0.728 & $0.005^{* * *}$ \\
\hline & $(0.695)$ & (1.028) & $(0.755)$ & $(1.690)$ & $(0.601)$ & $(1.276)$ & $(0.762)$ & $(0.804)$ & $(0.910)$ & $(0.747)$ \\
\hline \multirow{2}{*}{$\begin{array}{l}\text { Créditos al } \\
\text { consumo }\end{array}$} & $0.951^{* * *}$ & 0.000 & -0.383 & -0.000 & -0.244 & 0.000 & $0.558^{* * *}$ & 0.000 & $1.332^{* * *}$ & 0.000 \\
\hline & $(0.403)$ & $(0.206)$ & $(0.880)$ & $(0.668)$ & $(0.282)$ & $(0.329)$ & $(0.167)$ & $(0.325)$ & $(0.356)$ & $(0.233)$ \\
\hline
\end{tabular}

${ }^{* * *} \mathrm{p}<0.01,{ }^{* *} \mathrm{p}<0.05,{ }^{*} \mathrm{p}<0.1$. Errores estándar entre paréntesis. En cursiva los coeficientes cuando no se rechaza la hipótesis nula de la prueba de Hausman, en favor de RE. 
Dada la heterogeneidad de los municipios en términos de características fijas o con poca variación a través del tiempo, como su ubicación geográfica e intensidad migratoria, a continuación analizamos si existen efectos diferenciados entre regiones. ${ }^{12}$ Para tal fin, en la Tabla 5 presentamos únicamente los coeficientes estandarizados de la variable remesas. En general, los resultados son similares a los obtenidos con todos los municipios en cuanto a dirección y significancia estadística. En particular, en ninguna región se observa que, como resultado de las remesas, se genere un incremento de las sucursales bancarias o una reducción de las sucursales de las EACP. Esto es, las remesas están ayudando a transformar el rostro del sistema de intermediación financiera. Las diferencias principales entre regiones se encuentran en la magnitud del parámetro, destacando lo que ocurre en la región Sur. Con excepción de lo que acontece con las sucursales bancarias, en esta región todos los puntos de acceso y productos financieros que ofrecen la banca y EACP experimentaron alzas debido a las remesas, en mayor proporción que en el resto de las regiones. Es importante resaltarlo, pues es la zona con mayor tasa de pobreza (61\%) y exclusión financiera.

\section{Conclusión}

Diversos trabajos empíricos han estudiado la posible causalidad entre la recepción de remesas y la inclusión financiera. Sin embargo, hasta hoy no hay consenso en dicha relación. En este trabajo contribuimos a la literatura actual en distintos aspectos, tales como una estimación Poisson con variables instrumentales, el uso de distintas variables para la medición de la inclusión financiera, la incorporación de las entidades de ahorro y crédito popular, el uso de datos panel al nivel municipal, y la construcción de instrumentos con datos anteriormente no considerados. Si bien para la mayoría de los casos la estimación Poisson no contribuyó a incorporar a nuestros cálculos un número importante de municipios, debido a que muchos no presentan cambio en la variable dependiente durante todo el periodo estudiado, el uso de distintas metodologías y formas de definir las medidas de inclusión financiera contribuyen a verificar la robustez de nuestros hallazgos.

Los resultados indican que a mayores montos de remesas recibidos en los municipios hay un incremento - tanto para bancos como para las EACP- en el uso de

${ }^{12}$ Optamos por hacer las estimaciones por región, pues ya que la mayoría de las estimaciones se hicieron con efectos fijos, una variable que señalara la región no aportaría información adicional porque no cambia a través del tiempo. 
productos y servicios financieros. La única excepción es con relación al crédito hipotecario que ofrece la banca. Si bien las remesas podrían reducir las restricciones financieras de los clientes de los bancos y con ello reducir la demanda por dicho producto bancario, es también posible que este sea un dańo colateral causado por la estrategia bancaria de sustituir sucursales por corresponsales. Además, si agrupamos a los municipios en regiones geográficas se detecta que las remesas tienen un mayor impacto en el uso de productos financieros en los municipios del sur del país — espacio donde se registra la mayor tasa de pobreza en México-.

Si las remesas conducen a un mayor uso de productos crediticios, podría suponerse que - asumiendo una mora controlada — la rentabilidad que obtienen las instituciones financieras sería mayor. Ante ello, podría haber un mayor interés en mantener $u$ abrir sucursales con el fin de agilizar la firma de nuevos contratos crediticios. Sin embargo, esto no ocurre para el caso de las sucursales bancarias, lo cual es un resultado opuesto al que reportan Martínez Pería et al (2008), Demirgüç-Kunt et al. (2011) y Li Ng et al. (2015). No tenemos la suficiente información para responder a qué obedece este impacto negativo de las remesas. Puede ser resultado de un costo operativo que genera un bajo excedente, o porque las personas que reciben las remesas perciben mejores condiciones y trato con las EACP (Hernández y Martínez, 2021).

Los resultados encontrados aquí sugieren que las remesas pueden ayudar a elevar la inclusión financiera. Sin embargo, para que dicho incremento se traduzca en un mayor bienestar, es necesario que las personas puedan contar con herramientas que le permitan pagar y recibir dinero, así como ahorrar sin verse castigados de manera cotidiana por la inflación. Además, que existan los productos financieros que permitan a las personas atender sus necesidades de financiamiento. En este sentido, queda para futuras líneas de investigación analizar cuál de estos dos tipos de instituciones (bancos o EACP) ofrecen las mejores condiciones para lograr una inclusión financiera más cabal para las personas de bajos ingresos. 


\section{Referencias}

Aggarwal, R., Demirgüç-Kunt, A. y Martínez Pería, M. S. (2011). Do remittances promote financial development? Journal of developmenteconomics, 96 (2), 255-264.

Alliance for Financial Inclusion (AFI) (2016). Indicators of the Quality Dimension of Financial Inclusion. Guideline Note núm. 22. https://www. afi-global.org/publications/guideline-note-22-indicators-of-the-quality-dimension-of-financial-inclusion/

Allison, P. D., Williams, R. y Moral-Benito, E. (2017). Maximum likelihood for cross-lagged panel models with fixed effects. Socius, 3.

Anzoategui, D., Demirgüç-Kunt, A. y Martínez Pería, M. S. (2014). Remittances and financial inclusion: evidence from El Salvador. World Development, 54 (C), 338-349.

Ambrosius, C. (2011). Remittances and financial sector development. Lessons from the Salvadoran case. Savings and Development, 35 (1), 1-27.

Azizi, S. (2020). Impacts of remittances on financial development. Journal of Economic Studies, 47 (3), 467-477.

Banco de México. (2021). Ingresos por remesas. https://www.banxico.org.mx/ SieInternet/consultarDirectorioInternetAction.do?accion=consultarCuadro\&idCuadro $=$ CE81\&locale $=$ es .

Ben Naceur, S., Chami, R. y Trabelsi, M. (2020). Do remittances enhance financial inclusion in LMICs and in fragile states? IMF Working Paper, 2020 (066), https://doi.org/10.5089/9781513545394.001

Brown, R. P. y Carmignani, F. (2015). Revisiting the effects of remittances on bank credit: a macro perspective. Scottish Journal of Political Economy, 62 (5), 454-485.

Bureau of Economic Analysis. (2020). Data by place. https://www.bea.gov/ data/by-place-us.

Centro de Estudios Espinosa Yglesias (2018). Movilidad en México: las cinco regiones. https://ceey.org. mx/movilidad-social-en-mexico-las-cinco-regiones/.

Chibba, M. (2009). Financial inclusion, poverty reduction and the millennium development goals. The European Journal of Development Research, 21 (2), 213-230. 
Comisión Federal de Electricidad. (2018). Usuarios de consumo de electricidad por municipio (2010-2017). https://datos.gob.mx/busca/dataset/usuarios-y-consumo-de-electricidad-por-municipio-2010-2017

Comisión Federal de Electricidad. (2019). Usuarios y consume de electricidad por municipio (A partir de 2018). https://datos.gob.mx/busca/dataset/ usuarios-y-consumo-de-electricidad-por-municipio-a-partir-de-2018

Comisión Nacional Bancaria y de Valores. (2020). Portafolio de información. https://www.cnbv.gob.mx/Paginas/PortafolioDeInformacion.aspx

Demirgüç-Kunt, A., Córdova, E. L., Pería, M. S. M. y Woodruff, C. (2011). Remittances and banking sector breadth and depth: Evidence from Mexico. Journal of Development Economics, 95 (2), 229-241.

Glytsos, N.P. (2005). The Role of migrant remittances in development: evidence from Mediterranean countries. International Migration, 40(1), 5-26.

Giuliano, P., \& Ruiz-Arranz, M. (2009). Remittances, financial development, and growth. Journal of development economics, 90(1), 144-152.

Hsiao, C. (2007). Panel data analysis - advantages and challenges. Test, $16(1), 1-22$.

Hernández, F y A. Martínez (2021). The Dark Road to Credit Applications: The Small-Business Case of México. Journal of Financial Services Research, 1-25.

Kim, J. H. (2016). A study on the effect of financial inclusion on the relationship between income inequality and economic growth. Emerging Markets Finance and Trade, 52 (2), 498-512.

Li Ng, J. J. L., Sánchez Salinas, J. C. S., Hoyo Martínez, C. D. C. H. y Ramírez García, T. R. (2015). ¿La recepción de remesas tiene efectos sobre la inclusión financiera en México? Sobre México Temas de Economía (1).

Lin, W. y Wooldridge, J. (2019). Testing and Correcting for Endogeneity in Nonlinear Unobserved Effects Models. https://doi.org/10.1016/B9780-12-814367-4.00002-2

Martínez Pería, M. S., Mascaró, Y. y Moizeszowicz, F. (2008). Do remittances affect recipient countries' financial development? Remittances and Development: Lessons from Latin America [The International Bank for Reconstruction and Development / The World Bank], 171-215.

Secretaría de Relaciones Exteriores. (2020). Estadisticas de matrículas depersonas mexicanas en Estados Unidos. https://www.gob.mx/ime/acciones-y-programas/mexicanos-en-eua 